\title{
SAÚDE DO IDOSO: REFLEXÕES ACERCA DA INTEGRALIDADE DO CUIDADO
}

\author{
Elderly health: some thoughts on the care completeness
}

Artigo Original

\section{RESUMO}

Objetivo: Compreender na ótica do profissional, a prática no cuidado ao idoso, visando à integralidade. Métodos: Foi orientado pela pesquisa qualitativa, sendo utilizada para a coleta de dados a entrevista projetiva, tendo como disparador o uso de vinheta. Foi utilizada a técnica de análise de conteúdo, na modalidade temática. Os participantes foram 34 profissionais de duas unidades de Saúde da Família e seus Núcleos de Apoio à Saúde da Família correspondente. Resultados: O estudou revelou que existem avanços no cuidado ao idoso, contudo, identifica-se necessidade de aprimorar o processo de trabalho entre profissionais, pois o modelo curativo ainda é hegemônico. A escuta e o vínculo foram identificados como um instrumento indispensável para o alcance da integralidade. Conclusão: No espaço macro, foi possível identificar a necessidade de investimentos nos profissionais, como a Educação Permanente. É fundamental que os gestores estruturem uma rede de cuidado que inclua a pessoa idosa.

Descritores: Idoso; Integralidade em saúde; Estratégia Saúde da Família; Atenção Primária à Saúde; Envelhecimento; Gestão em Saúde.

\section{ABSTRACT}

Objective: The objective was to understand, on the professional point of view, the elderly care practice aiming completeness. Methods: The methodological approach was guided by qualitative research, with projective interview being used for data collection, having as a trigger the use of vignette. It was used as a methodological reference the content analysis, thematic modality. The subjects are 34 professionals of two family health units and their corresponding Support Family Health Centers. Results: The study shows that there is some progress in elderly care; however, it identifies the need to improve the work process among professionals because the treatment model is still hegemonic. Listening and bonding are identified as essential instrument for achieving completeness. Conclusion: In macro space, it was possible to identify the need for investment in professionals such as Continuing Education. It is also essential for managers to structure a care network that includes the elder.

Descriptors: Aged; Integrality in health; Family Health Strategy; Primary Health Care; Aging; Health management.

\author{
Simone de Carvalho Santos ${ }^{(1,2)}$ \\ Sílvia Franco da Rocha \\ Tonhom $^{(2)}$ \\ Ricardo Shoiti Komatsu(2)
}

1) Secretaria Municipal de Saúde de Marília - SMS - Marília (SP) - Brasil

2) Faculdade de Medicina de Marília FAMEMA - Marília (SP) - Brasil
Este artigo seguiu as normas e formatação estabelecidas pelo $5^{\circ}$ CIAIQ - Congresso Ibero-Americano em Investigação Qualitativa. 


\section{INTRODUÇÃO}

O envelhecimento da população é um dos maiores triunfos da humanidade e também um dos nossos grandes desafios $^{(1)}$. Em todo o mundo, a proporção de pessoas com 60 anos ou mais está crescendo rapidamente em comparação com outras faixas etárias. Em 2025, a previsão é que existirão aproximadamente 1,2 bilhões de pessoas com mais de 60 anos no mundo ${ }^{(1)}$.

Segundo o Instituto Brasileiro de Geografia e Estatística (IBGE), em 2010 a população de idosos era de 20.590.599, correspondente a $10,8 \%$ da população brasileira ${ }^{(2)}$. Estimase que em 2025 o Brasil representará cerca de 32 milhões de pessoas com 60 anos ou mais. Em 2050 a população idosa alcançará $22,71 \%$ da população total ${ }^{(3)}$. Um país já é considerado "velho" quando $7 \%$ da população é constituída por idosos ${ }^{(4)}$.

Dados mostram que são necessários investimentos na promoção da autonomia e da vida saudável dos idosos, assim como prover planejamento, logística para uma atenção adequada às suas necessidades ${ }^{(5)}$.

Envelhecer não significa estar ou ser doente. Mas significa que cuidados diferenciados devem ser oferecidos a essa população. Pois chegar à velhice remete às mudanças, que podem ser isoladas ou não. Sabe-se que o envelhecimento saudável e com qualidade, depende de inúmeros fatores, como por exemplo, sexo, genética, estilo de vida, alimentação, prática de atividade física.

Esse é um ponto primordial, pois muitos profissionais da saúde acreditam que envelhecer, sempre deve estar atrelado às doenças e ou limitações e como consequência esses profissionais não conseguem ampliar o olhar pensando na prevenção e promoção à saúde para essa população.

Promover a saúde do idoso significa considerar variáveis de distintos campos do saber, numa atuação interdisciplinar e multidimensional ${ }^{(6)}$.

Para a Saúde Pública, o envelhecimento tem se apresentado como um grande desafio. As mudanças na pirâmide populacional, que vai alargando seu ápice numa média de $2,5 \%$ de crescimento anual, geram preocupações para o sistema de saúde, porque mesmo sem ter solucionado os problemas sanitários relativos à infância, à adolescência e aos trabalhadores, terão de se equipar para dar respostas eficientes relativas à prevenção de enfermidades e à atenção aos idosos ${ }^{(4)}$.

A Atenção Primária à Saúde (APS) deve planejar, ofertar e executar ações de saúde em nível individual e coletivo, contemplando a promoção e proteção da saúde, a prevenção de agravos, o diagnóstico precoce e o tratamento de doenças, a reabilitação e a manutenção da saúde ${ }^{(7)}$.
Elaborar ações estratégicas para os idosos e colocálas em prática é um dos grandes desafios a ser enfrentados pelas Políticas de Saúde Pública, uma vez que estas ações dependem de um olhar integral para que possam proporcionar uma melhor qualidade de vida para os idosos.

Observa-se que o Sistema Único de Saúde (SUS) vem investindo em políticas públicas, pela busca de um olhar ampliado ao idoso, adotando estratégias para uma melhor estruturação, apontando mudanças e inovação no modelo de atenção, tendo como ponto de partida a APS.

Outro desafio do SUS é o de estimular os trabalhadores de saúde, inovando o modelo de atenção, reorganizando os serviços, pensando em estratégias que possam suprir as necessidades da população em sua integralidade.

A integralidade tem sido de difícil execução e garantia efetiva para a população, tendo em vista que demanda mudanças na concepção de trabalhos dos profissionais, onde as ações curativas permanecem completamente dissociadas da promoção e prevenção da saúde ${ }^{(8)}$.

A luta pela integralidade implica em repensar aspectos importantes na organização do processo de trabalho, gestão, planejamento e construção de novos saberes e práticas em saúde ${ }^{(9)}$.

Para conseguir operar satisfatoriamente a integralidade, nos campos de necessidades de saúde, o espaço da micropolítica do trabalho em saúde, aparece com evidência na medida em que este é um lugar por excelência de encontro entre os sujeitos trabalhadores e usuários, portanto, se dá o agir no cotidiano do cuidado à saúde ${ }^{(10)}$.

O trabalho em saúde pauta-se pelo referencial simbólico: ato de cuidar da vida, visto que o trabalhador opera um núcleo tecnológico no seu processo de trabalho, composto por trabalho morto e trabalho vivo. O trabalho vivo é o trabalho em ato, no seu exato momento de produção, na atividade do trabalhador. Já o trabalho morto é um trabalho já realizado, ou seja, resultado de um trabalho humano anterior. Afirmam ainda que mesmo ofertando os recursos tecnológicos necessários no cuidado, como exames de alta tecnologia, o processo de trabalho deve ter no seu núcleo de cuidado a hegemonia do trabalho vivo ${ }^{(10)}$.

Frente às necessidades de saúde, é necessário se dispor de todos os recursos tecnológicos disponíveis para a produção do cuidado, principalmente na micropolítica, onde o encontro entre o profissional e paciente é o melhor caminho para a gestão do cuidado. É primordial que o cuidado não se caracteriza apenas como um produtor de procedimentos e sim que seja uma linha de cuidado. Não é possível fazer cuidado em saúde se não dispor inicialmente da escuta entre os atores envolvidos.

Diante da necessidade de uma reformulação do sistema, surge em 1994 o Programa Saúde da Família (PSF), como 
um novo modelo de atenção de cuidado a saúde. Nasce como uma proposta para reestruturação do sistema de saúde no Brasil, embasados nos princípios e diretrizes do SUS ${ }^{(11)}$.

O PSF apresentou-se como uma possível mudança no modelo assistencial, buscando uma dinâmica de atuação das práticas de saúde que desse conta de avançar para a integralidade das ações de saúde.

Após duas décadas de sua implementação tem sido levantada, em diversos lugares e por diferentes atores sociais do SUS, uma hipótese da fragilidade do PSF. Parece haver um sentimento, mais ou menos difuso, de desencanto com o PSF, seja porque seria um modelo que não se aplica a todos os aspectos da realidade brasileira seja porque seria um modelo caro e pouco resolutivo ${ }^{(12)}$.

O PSF não fracassou, mas o ciclo da atenção básica à saúde, no qual ele nasceu e cresceu, esgotou-se. A saída não está em retroceder, mas em avançar, transformando-o de fato, numa Estratégia de Saúde da Família (ESF). Para isso, foi imprescindível diagnosticar os problemas existentes para poder superá-los ${ }^{(12)}$.

Sabe-se que as diferentes formas de organização local da ESF são determinantes para garantir a qualidade do atendimento. Alguns desafios importantes para a constituição de qualidade, organizadora da rede integrada de cuidados, são, além da fixação de profissionais qualificados nas equipes de Saúde da Família, o grau de efetividade clínica das equipes e a coordenação do cuidado individual com garantia da sua integralidade ${ }^{(7)}$.

Neste contexto, em $2008^{(13)}$ surge o Núcleo de Apoio à Saúde da Família (NASF) como um trabalho em saúde evolutivo, um marco importante na ampliação das possibilidades da ESF.

O NASF é uma equipe com profissionais de diferentes áreas de conhecimento e atua juntamente com os profissionais das equipes de Saúde da Família, compartilhando e apoiando as práticas em saúde nos territórios sob responsabilidade dessas equipes. A organização dos processos de trabalho dos NASF deve ter como foco o território sob sua responsabilidade, e deve ser estruturada priorizando o atendimento compartilhado e interdisciplinar, com troca de saberes, capacitação e responsabilidades mútuas, gerando experiência para todos os profissionais envolvidos, buscando a superação da fragmentação do cuidado existente no sistema ${ }^{(14)}$.

Consolidar a ESF como a estratégia de organização do SUS, significa entendê-la como parte e como centro de comunicação da Rede de Atenção a Saúde (RAS), exercitando os atributos essenciais e derivados da Atenção Primária $^{(12)}$.

As RAS são sistemas integrados de atenção à saúde, organizadas por meio de um conjunto, coordenado por pontos de atenção à saúde que tem no centro da comunicação a APS. Essa organização em rede é um caminhar para enfraquecer o sistema fragmentado de atenção à saúde ${ }^{(15,16)}$.

Baseado nos contextos acima discutidos pode afirmar que a APS é uma rede de atenção em construção, com aumento gradativo das equipes de Saúde da Família e a inclusão das equipes do NASF, que representam um marco diferencial nas possibilidades de melhores resultados indo de encontro com a integralidade do cuidado.

Perante as políticas de saúde voltadas à população idosa, esperamos que profissionais da ESF caminhem para a integralidade do cuidado. Devem estar sensibilizados, capacitados e instrumentalizados a identificar e atender às necessidades de saúde dessa população, promovendo assim uma vida mais saudável, minimizando, se possível, o tempo de incapacidades relacionadas à idade.

Atuar na Estratégia Saúde da Família permite-nos avanços frente às políticas de saúde propostas.O grande desafio de mudança a ser repensado é romper com a lógica da fragmentação da assistência para a integralidade do cuidado, sair de um modelo curativo, médico centrado, para um modelo de promoção e prevenção da saúde, baseado em um trabalho multidisciplinar e interdisciplinar.

Diante desses desafios pergunto: Os profissionais da ESF oferecem o cuidado ao idoso com ênfase na integralidade do cuidado? Optou-se, portanto, estudar o cuidado oferecido ao idoso a partir da visão dos profissionais da ESF.

O objetivo deste trabalho foi compreender na ótica do profissional a prática no cuidado ao idoso, visando a integralidade.

\section{MÉTODOS}

Trata-se de uma pesquisa descritiva, com intuito de descrever os fatos e fenômenos de determinada realidade ${ }^{(17)}$. Foi utilizado a abordagem qualitativa. $\mathrm{O}$ estudo qualitativo pareceu ser o mais adequado para o desenvolvimento deste projeto, pois permite avaliar a percepção, compreensão e valores dos profissionais no cotidiano de trabalho em relação ao cuidado ao idoso na $\operatorname{ESF}^{(18)}$.

A pesquisa foi realizada em um município do interior do estado de São Paulo, com uma população estimada, no ano de 2015 , de 232.006 habitantes $^{(19)}$, sendo 33.464 $\operatorname{idosos}^{(20)}$.

Os participantes da pesquisa foram os profissionais de duas equipes da ESF e seus respectivos NASFs. Como critério de inclusão, considerou-se as unidades com maior número de idosos da área de abrangência.

O universo dos participantes deste estudo foram 34 profissionais, dos quais 23 profissionais são das duas USF: 
três enfermeiros $(9 \%)$, dois dentistas $(6 \%)$, dois médicos $(6 \%)$, três auxiliares de enfermagem $(9 \%)$, dois auxiliares de saúde bucal (6\%), um auxiliar de limpeza (3\%), nove agentes comunitários de saúde $(25 \%)$ e um auxiliar de escrita (3\%); os outros onze participantes são dos dois NASF correspondentes: dois psicólogos (6\%), um terapeuta ocupacional (3\%), dois assistente social $(6 \%)$, um educador físico $(3 \%)$, dois nutricionistas $(6 \%)$ e três fisioterapeutas $(9 \%)$.

Como meio de comunicação entre o entrevistado e o entrevistador foi realizado uma entrevista projetiva, que possibilita utilizar dispositivos visuais, permitindo ao entrevistado discorrer sobre o que vê ou lề $\hat{e}^{(21)}$.

Como estratégia nesta pesquisa, foi utilizada vinhetas, disparadoras das entrevistas -que são histórias geradas a partir de uma variedade de fontes, permitindo-ao entrevistado referenciar fatores importantes no estudo das percepções, crenças e atitudes ${ }^{(22)}$. As vinhetas consistem em uma técnica de coleta de dados ideal para ser utilizada em pesquisas qualitativas, que queiram provocar o posicionamento dos participantes a partir de determinadas situações, servindo como um disparador de reflexões, de maneira que possibilite aos pesquisadores um diálogo mais próximo da ideia de pesquisa qualitativa ${ }^{(23)}$.

Foi utilizada como referencial metodológico, a análise de conteúdo. Enquanto método torna-se um conjunto de técnicas de análise das comunicações, não se tratando de um instrumento, mas sim de um método que utiliza procedimentos para interpretação do conteúdo das mensagens, permitindo inferência de conhecimentos ${ }^{(24)}$.

O projeto de pesquisa foi aprovado pelo Comitê de Ética em Pesquisa (CEP) sob o parecer de $\mathrm{n}^{\circ} 1.01 .846 \mathrm{e}$ pelo Comitê de Avaliação de Pesquisa da Secretaria da Saúde (COMAP), atendendo a resolução 466/2012.

\section{RESULTADOS E DISCUSSÃO}

\section{A Gestão do Cuidado a Pessoa Idosa no espaço Micro}

Cada profissional pode ter um olhar diferente de uma mesma situação, pois a forma de interpretar pode ser influenciada por experiências, vivências, necessidades e objetivos. Komatsu ${ }^{(25)}$ traz essa reflexão ao descrever que uma lente não é igual à outra, existe uma singularidade em cada olhar, resultante de sua vivência, experiência e ponto de vista.

As vinhetas proporcionaram uma visão das múltiplas facetas do cuidado ao idoso, trazidas pelos relatos dos profissionais da ESF e NASF. Nos discursos, os participantes reconhecem que o cuidado ao idoso perpassa pelo contexto familiar, conforme observado nas falas abaixo:
"[...] trabalhar de uma forma integral esse contexto familiar. Procurar trabalhar a base familiar, família rachada é sinônimo de doença fisisica e doença emocional, família estruturada na base com uma boa relação familiar é o primeiro passo para a gente conseguir promover a saúde e não curar a doença. Então tem que trabalhar a união familiar, trabalhar a integralidade." (MED 2)

Podemos destacar a importância da família no cuidado ao idoso, pois é no reduto desse espaço que o envelhecimento ganha significância e magnitude. O idoso e família requerem atenção especialmente dos profissionais de saúde, para que o cuidado oferecido seja de qualidade e humanizado ${ }^{(26)}$.

Para Cecílio(27), podemos definir a gestão do cuidado de acordo com as necessidades de saúde singulares de cada pessoa e família, visando o bem-estar, segurança e autonomia. Trata-se da gestão do cuidado que tem como atores privilegiados: pessoas da família.

A ESF tem como uma de suas responsabilidades "a família". A equipe deve ofertar cuidado ao idoso e sua família. É necessário que os profissionais visualizem o cuidado ao idoso no seu contexto familiar multidimensional, considerando sua cultura, religião, relações interpessoais, conflitos, cotidiano, vivências e experiências ${ }^{(28)}$.

Profissionais trouxeram em suas falas esse olhar voltado ao cuidado familiar, mas isso não é uma garantia de sua efetivação na prática diária. Este panorama do cuidado tem sido um grande desafio para a saúde, pois implica em abrir novos horizontes no ato de cuidar, significa necessidades de modificações no processo de trabalho, no cotidiano e na prática do dia a dia.

Olhar o idoso sem caracterizar e contextualizar sua família segue uma lógica inversa da integralidade. A família pode ser uma provedora de cuidados ao idoso, mas também deve ser "olhada" como sujeitos que demandam um cuidado em sua singularidade.

Ampliar o olhar para além do idoso é um grande desafio na integralidade do cuidado. Nota-se que os profissionais trouxeram em suas falas, além do olhar do idoso no seu contexto familiar, o olhar para o cuidador familiar.

\footnotetext{
"Como está a alimentação, dieta, e além de tudo ela é cuidadora, ela tem uma sobrecarga dos cuidados com a mãe e além desse cansaço de ser cuidadora [...]. Por ser cuidadora, tem um desgaste emocional, tem um desgaste fisico. "(ENF 1)

O cuidador de idosos geralmente é um familiar, do sexo feminino, e muitas vezes pode ser um idoso. Assumir e desempenhar o papel principal no suporte dessa assistência ao idoso pode ser por vontade, disponibilidade, identificação ou até mesmo por falta de opção.
} 
Frequentemente os familiares de idosos, que assumem o papel de cuidador, vão aos poucos sofrendo mudanças na atividade familiar e social. Podem apresentar fragilidades orgânicas e emocionais. Sentimentos diversos como raiva, cansaço, sofrimento, frustração e medo podem surgir no meio deste novo contexto. É evidente que o meio familiar em que o idoso vive, ou seja, os laços familiares influenciam de forma significativa nesse cuidado.

Para Cecílio ${ }^{(27)}$, dependendo da complexidade dos laços familiares, da sobrecarga de trabalho, das exigências permanentes para a realização do cuidado, a dimensão familiar muitas vezes pode ser conflituosa, principalmente entre cuidadores e cuidado.

A tarefa de cuidar de um idoso é muito desgastante, implica em riscos a saúde do cuidador. A equipe de saúde deve oferecer cuidados ao cuidador para que os mesmos não adoeçam, amparando suas necessidades e singularidades, pois as necessidades dessas pessoas podem ser diversas e distintas.

Minayo e Coimbra Júnior ${ }^{(4)}$ afirmam que o cuidador de idoso necessita de uma rede de apoio ampla, inclui desde consultas até o suporte estratégico, emocional e institucional para quem cuida. Quando os cuidadores contam com uma estrutura de apoio no cuidado, têm a possibilidade de exercer o cuidado e permanecer inseridos socialmente sem imobilizar-se pela sobrecarga determinada pela difícil e estafante tarefa.

Salienta-se um olhar especial dos profissionais para além do idoso que está sendo cuidado, ou seja, um olhar voltado ao cuidador, para que o cuidado oferecido seja de qualidade e humanizado. Torna-se assim possível pensar em estratégias que alcancem ou pelo menos minimizem as conseqüências da saúde/doença do cuidador.

Nesse sentido, para uma melhor compreensão do contexto familiar e individual, profissionais identifica a visita domiciliária (VD) como um instrumento importante que proporciona uma visão diferenciada da realidade vivenciada pelo idoso.

"Eu estaria indo até a dona Joana, fazendo visita, conhecendo a realidade 'in loco', como são as condições, estrutura da casa, condições sócio-econômica dela e ai partir propriamente para uma avaliação das condições fisicas dela, tendo em vista que ela já é uma paciente com doenças crônicas e provavelmente ela já recebia esses cuidados [...]." (FISIO 2)

Na visita domiciliária é possível estabelecer, junto aos familiares, um suporte mais adequado às necessidades específicas da pessoa idosa, negociando com familiares aspectos desse cuidado ${ }^{(29)}$. As visitas domiciliares possibilitam maior vínculo familiar, reunindo seus aspectos afetivos, culturais, religiosos e sociais.
O vínculo é um dos princípios da ESF. Consiste na construção de uma relação de confiança entre o paciente e profissional. A construção de vínculo possibilita corresponsabilização pela saúde. Permite uma melhor compreensão das necessidades de saúde do usuário, fomenta a efetividade das ações assistenciais, favorece a participação mais ativa do usuário, qualificando assim a assistência oferecida.

A visita domiciliária é uma estratégia importante na proposta de vigilância à saúde, porém deve-se ter cuidado para que não seja realizada apenas por uma necessidade de cobrança de metas, vigilância ou controle. Nota-se por meio dos discursos dos profissionais, que nem sempre a visita domiciliária é utilizada como uma ferramenta importante a somar no cuidado:

"O idoso tem que ser visto de forma diferente [...]. Não pode esperar chegar a esse ponto. Cadê o ACS que não viu isso em visita? [...]." (ACS 8)

A visita domiciliária é uma prática que tem sido substituída pela ação cotidiana dos agentes comunitários de saúde e ocasionalmente dos demais profissionais. O simples fato de realizá-las, não significa que o profissional reconheça suas potencialidades. ${ }^{(30,31)}$

Fazer parte prioritária da ESF não garante que a VD seja uma ferramenta potencializadora no processo de trabalho. Pode simplesmente fazer parte do cotidiano dos profissionais, como uma necessidade "cobrada" verticalmente para cumprimento de metas, sem enfoque na produção de cuidado e sem planejamento das prioridades. Podemos utilizar como exemplo o ACS, que perante o Ministério da Saúde, deve realizar VD mensais a todas as famílias de sua área de abrangência. Essa obrigatoriedade pode fazer com que o ACS não atue conforme as necessidades identificadas.

Para o avanço na gestão do cuidado ao idoso, os trabalhadores precisam caminhar para um "novo" paradigma de organização dos serviços, uma mudança no modelo de assistência. Nas falas dos entrevistados observamos que essas propostas ainda estão um pouco distante da realidade vivenciada, pois os profissionais reconhecem que muitas vezes o olhar ainda está voltado para o modelo fragmentado e curativo:

"Às vezes fica mais voltada à parte clínica [...] A gente não consegue fazer o antes, só faz quando está emergencial, e ai a gente continua no curativo [...]". (NUTRI 2)

Por que na ESF ainda permeia o olhar fragmentado, curativista, voltado às soluções rápidas e pontuais? $\mathrm{O}$ formato do trabalho da equipe da ESF não deveria garantir a ruptura centrada na doença? 
Ainda vivemos um SUS fragmentado, voltado ao atendimento às urgências, com necessidades de resultados em curto prazo. Nota-se que na prática diária na ESF, muitas atitudes são sustentadas no modelo curativo, centrado na doença, distanciando o cuidado integral como o foco de necessidade.

Para Franco e Merhy ${ }^{(31)}$, os princípios da ESF não garantem a forma ideal de produção de saúde, ou seja, no modo de como se produz cuidado a partir do cotidiano, nas relações estabelecidas entre os trabalhadores e usuários.

Podemos afirmar que mesmo após a implantação da ESF, a essência desse processo de trabalho não garante mudanças na prática do cuidado. O modelo de assistência ainda pode estar direcionado para uma vertente de tecnologia, ou seja, predomínio da tecnologia dura.

Os autores Merhy e Feuerwerker ${ }^{(32)}$ descrevem as tecnologias fazendo uma comparação com três tipos de caixas de ferramentas: a primeira é vinculada a propedêutica e aos equipamentos (diagnósticos e terapêuticos), essa caixa chamamos de tecnologia dura; a segunda permite processar o olhar do saber profissional, a essas chamamos de tecnologias leve-dura; já a terceira caixa de ferramenta é a que permite a produção de relações envolvidas no encontro trabalhador-usuário, mediante escuta, interesse, criação de vínculo, chamada de tecnologia leve.

Dependendo do uso dessas caixas de ferramentas, configuram-se distintos modelos de atenção à saúde. Observamos na prática diária que existe uma tendência ao uso das tecnologias duras e leve-duras, levando ao modelo queixa e conduta. Importante ressaltar que as tecnologias leves podem ser utilizadas por todos os trabalhadores, independente de sua especificidade profissional.

Trabalhar com a interface da micropolítica do processo de trabalho, nos remete subjetividade de cada profissional. Nesse sentido as equipes de saúde podem contribuir positivamente ou não para a produção do cuidado em saúde. O modo de como organizam e principalmente atuam o modelo de trabalho, é que conduzirá o modo de produzir saúde ao idoso ${ }^{(28)}$.

Nas entrevistas realizadas, profissionais reconhecem que são detentores de possibilidades de mudança e destaca o Plano Terapêutico Singular (PTS) como uma ferramenta importante na construção do cuidado:

"Eu acredito que um caso assim a gente teria que estar discutindo enquanto equipe [...] para a gente ver o plano terapêutico para essa família, né! [...] estar envolvendo todos os profissionais [...]. Para ver o olhar da equipe pra construção do plano terapêutico." (TO)

O Projeto Terapêutico é um plano de ação composto por intervenções que seguem uma intencionalidade visando o cuidado integral. Um PTS deve ser elaborado a partir de análise dos profissionais sobre as múltiplas dimensões do sujeito $^{(33)}$.

O PTS é um instrumento de trabalho que potencializa o profissional, estimula o grau de autonomia dos trabalhadores, proporciona maior articulação da equipe, permite uma qualificação do cuidado por meio de reflexões, discussões, compartilhamento de responsabilidades e ações. Outro ponto que merece destaque é que o PTS tem que ser construído em conjunto, com todos os profissionais envolvidos no caso e com o usuário, pois existe o risco do usuário em produzir sozinho as adaptações que achar pertinente.

Todavia é importante destacar que para um impacto positivo no processo de trabalho, o PTS tem que ser operador do uso das tecnologias leves, em relação às tecnologias leve-duras e dura.

\section{Reflexões da gestão do cuidado no macro espaço}

Pensar em processos de trabalho que combine cura, promoção e proteção, é um desafio dos gestores e trabalhadores. As definições dos fluxos na saúde dependem fundamentalmente de esforço e vontade política dos gestores $^{(10)}$.

Para Cecílio( ${ }^{(9)}$, a integralidade do micro reflete no macro e o macro reflete no micro. O processo de trabalho se opera no espaço da micropolítica ou no espaço macro. Independente do espaço a organização impacta no modo de se produzir saúde ${ }^{(9)}$. Por meio dos relatos observamos que existe fragilidade da gestão municipal na saúde do idoso.

"Com a secretaria temos pouco contato enquanto saúde do idoso, acho que ainda falta muita coisa, a secretaria não está estruturada [...] ainda não está efetivado." (ASOCIAL 2)

"Também faria uma discussão da saúde do idoso [...] Apesar de ser um serviço pouco estruturado no municipio." (ENF 2)

A dimensão sistêmica da gestão do cuidado é aquela que trata de construir conexões formais na perspectiva da construção da integralidade do cuidado. Os gestores têm uma responsabilidade intransferível na gestão sistêmica do cuidado em saúde ${ }^{(27)}$.

A programação do macro deveria acontecer embasada em cada equipe da ESF a partir das necessidades reais dos idosos, família e comunidade vinculadas às equipes. Por meio da programação embasada no micro, é possível pensar em intervenções de atenção secundária, terciária, ambulatoriais e hospitalares, além dos sistemas de apoio como a patologia clínica e assistência farmacêutica. É assim que se constrói uma ESF com capacidade de ser 
efetivamente uma estratégia de organização do SUS. Deve pensar em indicadores da programação e ser balanceado, especialmente, em termos de processos e resultados. Esses indicadores devem estar referidos nas prioridades da SMS expressas no Plano Municipal de Saúde. Ao longo do tempo, os indicadores podem variar, em função das prioridades políticas da $\mathrm{SMS}^{(12)}$.

A integralidade do cuidado ao idoso permeia na articulação do saber e fazer de cada profissional, de cada equipe e dos gestores. A gestão municipal não pode tentar caminhar sozinha, embasado apenas nas ações verticalizadas do Ministério da Saúde. Há necessidade de se criar e recriar, na gestão municipal da saúde do idoso, planos e investimentos de projetos e diretrizes embasadas nas políticas do idoso, que vá ao encontro com as possibilidades da ESF e necessidades dos idosos. Por isso é necessário que os gestores pensem em estratégias a partir das lentes da micropolítica e do envolvimento dos profissionais que prestam assistência direta aos idosos, para a construção da rede de cuidado do município.

A integralidade exige um esforço em entender o conjunto de saberes e práticas no cuidado à saúde. Ao mesmo tempo a integração de vários serviços tem sido uma dificuldade para gestores e usuários, em que estes têm o seu "caminhar na rede" dificultado por falta de articulação destes recursos ${ }^{(34)}$.

A intersetorialidade permite a articulação entre os diferentes setores, possibilita desenvolver projetos de melhor qualidade, que não seriam obtidos com ações isoladas. $\mathrm{Na}$ prática do dia a dia, profissionais da ESF e NASF vivenciam entraves na execução da intersetorialidade:

"A gente também tem dificuldades com as parcerias para a gente conseguir uma resolutividade [...]. Fica dificil buscar parcerias, porque às vezes eles acabam devolvendo o caso para saúde, principalmente os casos que a gente sabe que tem negligência com idosos, fica bem dificil [...].” (TO)

A Política Nacional de Saúde da Pessoa Idosa destaca em suas diretrizes o estímulo às ações intersetoriais, com vistas à integralidade da atenção $\mathrm{O}^{(35)}$.

Para a ESF é necessário a articulação com os programas de intersetorialidade, pois não dá para pensar em integralidade do cuidado se não evoluir para parcerias com outros setores. A construção da intersetorialidade possibilita descobertas de caminhos para ação, por meio de compartilhamentos de conhecimentos.

Embora a intersetorialidade seja uma das prioridades da PNI, é de suma importância que os gestores tenham como meta no seu plano municipal uma política abrangente que preconize a comunicação entre os diversos setores e que consigam sensibilizar os profissionais, que nem sempre sentem necessidade de compartilhamento do cuidado, caminham para uma construção da assistência focada apenas no setor saúde.

Outro ponto, que consideramos importante para a produção de mudança no processo de trabalho, é o investimento dos gestores referente aos recursos humanos. Ao discutir qualificação profissional, estamos dizendo em qualificar os serviços prestados à população idosa.

Percebe-se pouco "investimento" nos profissionais da ESF e NASF:

"[...] a gente fica com uma fragilidade por falta de capacitação e conhecimento [...]." (NUTRI 2)

Em um estudo foi demonstrado que existem lacunas na gestão da saúde do idoso, com necessidades de investimentos em capacitação dos profissionais, inclusão de agendas e protocolos para nortear o cuidado ao idoso em sua integralidade. ${ }^{(36)}$. Conforme se constata nos depoimentos, observa-se que não existe uma organização municipal frente à Educação Permanente (EP).

"[...] eu sinto falta em não ter educação permanente durante nossas reuniões de equipe [...] acho que nos ajuda muito". (ENF 2)

A desvalorização dos profissionais de saúde, as fragilidades nas relações de trabalho, o baixo investimento em processos de educação permanente e a pouca participação na gestão dos serviços, dificulta a consolidação no espaço da micropolítica ${ }^{(37)}$.

Sabe-se que a EP é uma estratégia fundamental para o funcionamento da prática em saúde com qualidade. Ela permite uma aproximação com os desconfortos e incertezas dos trabalhadores. O diálogo é um caminho para produção de mudanças na prática de saúde, para construção de protocolos, diretrizes, construção de "novos" pactos em saúde.

Baseado na PNAB, o processo de educação permanente potencializa enormemente o desenvolvimento de competências de gestão e de cuidado, na medida em que aumenta as alternativas para o enfrentamento das dificuldades vivenciadas pelos trabalhadores em seu cotidiano $^{(38)}$.

Sabemos que a mudança no trabalhador, a mudança no trabalho em saúde não é totalmente controlável, pois cada profissional tem seus valores, suas concepções, suas idéias acerca da saúde. Podemos afirmar que a EP é um caminho, é uma estratégia que possibilita construção de novos valores, reflexões, aprendizagem no trabalho, transformação na prática dos trabalhadores impactando no mundo tecnológico do trabalho. 
Desta maneira a gestão municipal deveria ter como uma das ações prioritárias a EP para os profissionais da APS, pois proporciona às equipes agregação de novos conhecimentos, tornando-as protagonistas dos processos de produção do cuidado ás necessidades de saúde de todos e, em especial, a dos idosos.

\section{CONSIDERAÇÕES FINAIS}

O fenômeno do envelhecimento populacional lança grandes desafios ao SUS, em especial para os profissionais da Atenção Básica, que é considerada o centro de comunicação entre os diversos pontos da rede.

A pesquisa proporcionou avaliar o cuidado oferecido ao idoso com ênfase na integralidade, no espaço micro e macro espaço de gestão.

Trabalhar com a interface da micropolítica do processo de trabalho, nos remete a subjetividade de cada profissional, especialmente no cenário da produção do cuidado. Profissionais reconhecem a importância da família no âmbito do cuidado ao idoso e, para tal, tanto o idoso como sua família necessita de atenção especial das equipes de saúde, para que o cuidado oferecido seja de qualidade e humanizado. A escuta e o vínculo são destaque para o alcance da integralidade. Nota-se que na assistência ainda permeia o modelo curativo, distanciando o cuidado em sua integralidade. $\mathrm{O}$ modo de como organizam e principalmente atuam o modelo de trabalho, é que conduzirá o modo de produzir saúde ao idoso.

No contexto do macro espaço de gestão, foi possível identificar a necessidade do planejamento das ações de forma conectada com as necessidades de cada território, no sentido de oportunizar mudanças necessárias a cada realidade de saúde. Profissionais identificam EP e a intersetorialidade como dispositivos imprescindíveis na produção do cuidado qualificado. Porém destacam fragilidade em incentivos e investimentos dos gestores.

Os gestores são atores fundamentais e responsáveis no processo de mudança da produção da saúde, e em razão disso devem elaborar dispositivos que contribuam para a criação de novas formas de relacionamento dos trabalhadores de saúde com os usuários.

A Atenção Primária precisa avançar no cuidado a pessoa idosa. Com o atual cenário do envelhecimento e toda a operacionalização do SUS, os gestores precisam incluir em suas prioridades um espaço consolidado para a saúde do idoso.

Sugere-se um repensar do processo de trabalho ofertado aos idosos pelos profissionais do macro e micro, para que não se repita o modelo de atenção desumanizado, fragmentado, centrado no biológico. Maior aproximação dos profissionais das equipes dos diferentes cenários de prática, da gestão e usuários, proporcionando espaços de diálogo, buscando construir caminhos para o cuidado em sua integralidade, em especial, ao idoso.

\section{REFERÊNCIAS}

1. Organização Mundial da Saúde. Envelhecimento ativo: uma política de saúde. Brasília (DF): Organização PanAmericana da Saúde Brasília; 2005.

2. IBGE. Sinopse do censo demográfico 2010 [Internet]. Rio de Janeiro (RJ): IBGE; 2011 [citado 10 jul 2014]. Disponível em: http://www.ibge.gov.br/home/ estatistica/populacao/censo2010/default_sinopse.shtm

3. Moraes EN. Atenção à saúde do idoso: aspectos conceituais. Atenção à saúde do idoso: aspectos conceituais. Brasília (DF): OPAS; 2012.

4. Minayo MCS, Coimbra Júnior CEA. Entre a liberdade e a depêndencia: reflexões sobre o fenômeno social do envelhecimento. In: Minayo MCS, Coimbra Júnior CEA, editores. Antropologia, saúde e envelhecimento. Rio de Janeiro (RJ): Fiocruz; 2002. p. 11-24.

5. Minayo MCS. O envelhecimento da população brasileira e os desafios para o setor saúde. Cad Saúde Pública. 2012;28(2):208-9.

6. Ciosak SI, Braz E, Costa MFBNA, Nakano NGR, Rodrigues J, Alencar RA, Rocha ACAL. Senescência e senilidade: novo paradigma na atenção básica de saúde. Rev Esc Enferm USP. 2011;45(Esp 2):1763-8.

7. Organização Pan-Americana da Saúde. Ministério da Saúde, Conselho Nacional de Secretários Estaduais de Saúde, Conselho Nacional de Secretários Municipais de Saúde. Inovando o papel da Atenção Primária nas redes de Atenção à Saúde: resultados do laboratório de inovação em quatro capitais brasileiras. Brasília (DF): Organização Pan-Americana da Saúde; 2011.

8. Albuquerque PC, Stotz EN. A educação popular na atenção básica à saúde no município: em busca da integralidade. Interface Comunic Saúde Educ. 2004;8(15):259-74.

9. Cecílio LCO. As necessidades de saúde como conceito estruturante na luta pela integralidade e equidade na atenção em saúde. In: Pinheiro R, Mattos RA, editores. Os sentidos da integralidade na atenção e no cuidado à saúde. Rio de Janeiro (RJ): ABRASCO; 2001. p. 113-26.

10. Merhy EE, Franco TB. Por uma composição técnica do trabalho centrada nas tecnologias leves e no campo relacional. Saúde Debate. 2003;27(65):316-23. 
11. Brasil. Ministério da Saúde. Programa Saúde da Família. Brasília (DF): COSAC; 1994.

12. Mendes EV. O cuidado das condições crônicas na atenção primária à saúde: o imperativo da consolidação da estratégia da saúde da família. Brasília (DF): Organização Pan-Americana da Saúde; 2012.

13. Brasil. Ministério da Saúde. Portaria $n^{\circ} 154$, de 24 de janeiro de 2008. Cria os Núcleos de Apoio à Saúde da Família - NASF. Diário Oficial da União, Brasília (DF); 25 jan 1994; Seção 1:47.

14. Brasil. Ministério da Saúde. Diretrizes do NASF: Núcleo de Apoio à Saúde da Família. Brasília (DF): Ministério da Saúde; 2010.

15. Mendes EV. As redes de atenção à saúde. Brasília (DF): Organização Pan-Americana da Saúde; 2011.

16. Mendes EV. A construção social da atenção primária à saúde. Brasília (DF): CONASS; 2015.

17. Gil AC. Como elaborar projetos de pesquisa. 5a ed. São Paulo (SP): Atlas; 2010.

18. Minayo MCS. O desafio do conhecimento: pesquisa qualitativa em saúde. $13^{\text {a }}$ ed. São Paulo (SP): Hucitec; 2013.

19. IBGE. Cidade@: Marília (SP) [Internet]. Rio de Janeiro (RJ): IBGE; 2016 [citado 27 jun 2016]. Disponível em: http://cidades.ibge.gov.br/xtras/perfil. php?lang $=\& \operatorname{codmun}=352900$

20. Fundação Sistema Estadual de Análise de Dados SEADE. Projeções populacionais: Marília [Internet]. São Paulo (SP): SEADE; 2014 [citado 17 nov 2014]. Disponível em: http://produtos.seade.gov.br/produtos/ projpop/

21. Minayo MCS. Trabalho de campo: contexto de observação, interação e descoberta. In: Minayo MCS, organizadora. Pesquisa social: teoria, método e criatividade. 32a ed. Petrópolis (RJ): Vozes; 2012. p. 61-78.

22. Hughes R. Considering the vignette technique and its application to a study of drug injecting and HIV risk and safer behaviour. Sociol Health Illn. 1998;20(3):381400.

23. Gourlay A, Mshana G, Birdthistle I, Bulugu G, Zaba $\mathrm{B}$, Urassa $\mathrm{M}$. Using vignettes in qualitative research to explore barriers and facilitating factors to the uptake of prevention of mother-to-child transmission services in rural Tanzania: a critical analysis. BMC Med Res Methodol. 2014;14:21.
24. Bardin L. Análise de conteúdo. São Paulo (SP): Edições 70; 2012.

25. Komatsu RS. Aprendizagem baseada em problemas na Faculdade de Medicina de Marília: sensibilizando o olhar para o idoso [tese]. Marília (SP): Universidade Estadual Paulista; 2003. 234 p.

26. Silva KM, Santos SMA, Souza AIJ. Reflexões sobre a necessidade do cuidado humanizado ao idoso e família. Saúde Transform Soc. 2014;5(3):20-4.

27. Cecilio LCO. Apontamentos teórico-conceituais sobre processos avaliativos considerando as múltiplas dimensões da gestão do cuidado em saúde. Interface Comun Saúde Educ. 2011;15(37):589-99.

28. Santos SC, Tonhom SFR, Komatsu RS. Integralidade do cuidado ao idoso na Estratégia Saúde da FamiliaESF. In: Atas $5^{0}$ Congresso Ibero-Americano em Investigação Qualitativa em Saúde , $1^{\circ}$ International Symposium on Qualitative Research; 2016; V 2. p.1292-1301.

29. Brasil. Ministério da Saúde. Envelhecimento e saúde da pessoa idosa. Brasília (DF): Ministério da Saúde; 2006.

30. Merhy EE, Malta DC, Santos FP. Desafios para os gestores do SUS, hoje: compreender os modelos de assistência à saúde no âmbito da reforma sanitária brasileira e a potência transformadora da gestão. In: Freese E, organizador. Municípios: a gestão da mudança em saúde Recife: UFPE; 2004. p.45-76.

31. Franco TB, Merhy EE. Programa de Saúde da Família (PSF): contradições de um programa destinado à mudança do modelo tecnoassistencial. In: Merhy EE, Magalhães Júnior HM, Rimoli J, Franco TB, Bueno WS, organizadores $\mathrm{O}$ trabalho em saúde: olhando e experienciando o SUS no cotidiano. São Paulo (SP): Hucitec. 2003. p. 55-124.

32. Merhy EE, Feuerwerker LCM. Novo olhar sobre as tecnologias de saúde: uma necessidade contemporânea. In: Mandarino ACS, Gomberg E, organizadores. Leituras de novas tecnologias e saúde. São Cristóvão (BA): UFS, UFBA; 2009. p.29-74.

33. Brasil. Ministério da Saúde. Saúde mental. Brasília (DF): Ministério da Saúde; 2013.

34. Franco TB, Mehry EE. Trabalho, produção do cuidado e subjetividade em saúde. São Paulo (SP): Hucitec; 2013.

35. Brasil. Ministério da Saúde. Política Nacional de Promoção da Saúde: documento para discussão. Brasília (DF): Ministério da Saúde; 2002. 
36. Fernandes MTO. A rede de atenção à saúde para o cuidado à pessoa idosa na atenção primária: um modelo teórico [tese na Internet]. Belo Horizonte (MG): Universidade Federal de Minas Gerais; 2013. 209 p.[citado 20 jan 2016]. Disponível em: http:// www.bibliotecadigital.ufmg.br/dspace/bitstream/ handle/1843/GCPA-99PQ4Y/maria_teresinha_de oliveira_fernandes.pdf? sequence $=1$

37. Brasil. Conselho Nacional de Secretários de Saúde. Planificação da atenção primária à saúde nos estados. Brasília (DF): CONASS; 2011.
38. Brasil. Ministério da Saúde. Política Nacional de Atenção Básica. Brasília (DF): Ministério da Saúde; 2012.

\section{Endereço para correspondência:}

Simone de Carvalho Santos

Av José de Grande, 332

CEP: 17519-470 - Marília - SP - Brasil

E-mail: simonezihlmam@gmail.com 